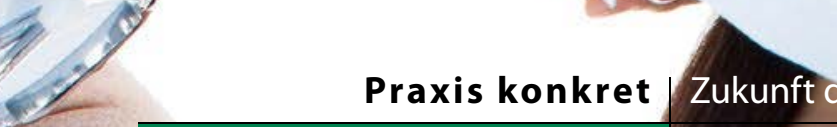

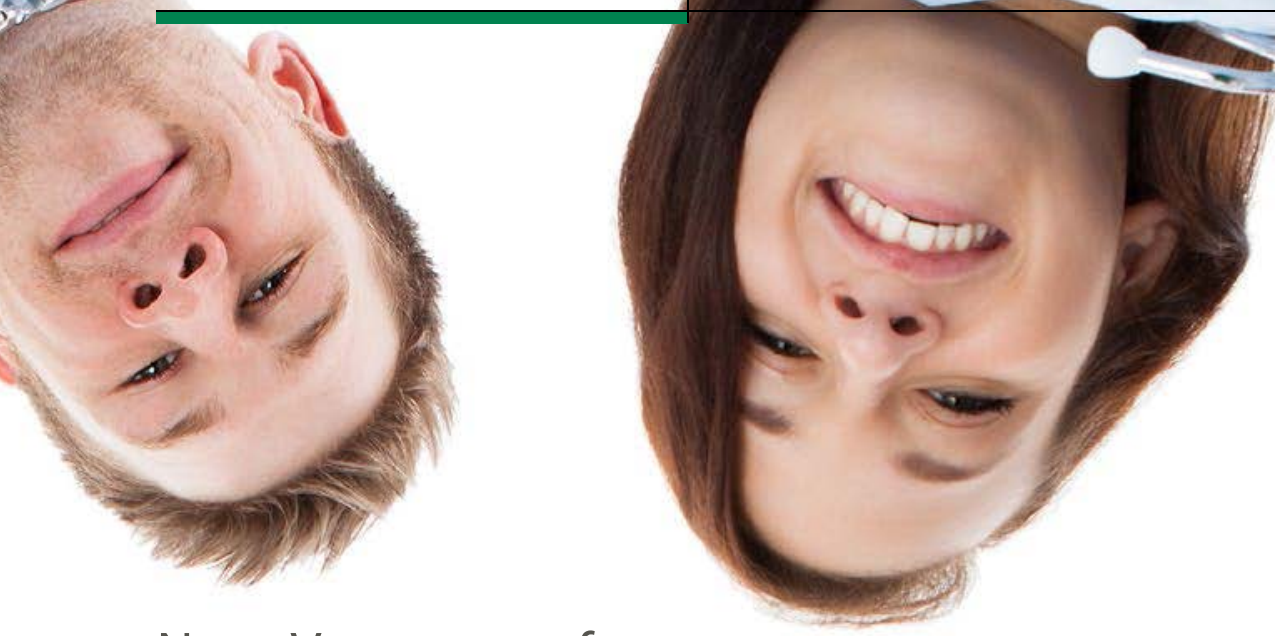

Neue Versorgungsform

\section{Auch Privatpatienten sollen von ASV profitieren}

\author{
Die ambulante spezialfachärztliche Versorgung (ASV) scheint langsam \\ Fahrt aufzunehmen. Der Vorsitzende des ASV-Bundesverbandes wirbt \\ dafür, auch Privatpatienten an der neuen Versorgungsform teilhaben \\ zu lassen.
}

A uch wenn die ASV zunächst nur ein Angebot für gesetzlich Versicherte ist: Die privaten Krankenversicherer sollten den Sektor genau im Auge behalten, um auch ihren Kunden entsprechende Lösungen anbieten $\mathrm{zu}$ können. Davon geht Axel Munte aus, der Gründer und Vorstandsvorsitzende des Bundesverbands ASV. „Die rund 10\% Privatversicherten müssen die Chance haben, an einer Strukturverbesserung für Schwerstkranke partizipieren zu können“, sagte Munte bei einem Symposium zur medizinischen Versorgung des privaten Krankenversicherers DKV in Köln. Wenn sich ASV-Teams etabliert haben und gute Versorgungsergebnisse erzielen, werden die PKV-Versicherten entsprechende Angebote einfordern, erwartet Munte. Wann die Unternehmen ihre Kunden über das neue Angebot informieren und auf spezialisierte Teams hinweisen sollten, sei im Moment aber noch nicht abzusehen.

\section{Gemeinsame Betreuung im Fokus}

Der ehemalige Vorsitzende der KV Bayerns setzt große Hoffnungen in die ASV.
„Ich würde so etwas in meinem Alter nicht mehr anfassen, wenn ich darin nicht eine Riesenchance sehen würde." Nach Informationen der ASV-Servicestelle gab es Mitte Oktober bereits 23 ASV-Teams, davon zwölf für gastrointestinale Tumoren und elf für die Indikation Tuberkulose. Im Vergleich: Im November 2014 waren es noch sechs Teams. Der Bereich befinde sich in stetiger Entwicklung, sagte er. Am Ausbau der interdisziplinären und intersektoralen Kooperation führt nach Muntes Einschätzung kein Weg vorbei. „Patienten mit schweren Krankheiten werden von den besten Ärzten ambulant und stationär gemeinsam im Team betreut", skizzierte er das ehrgeizige Ziel der ASV. Dafür sei es wichtig, dass die Mediziner aus beiden Sektoren tatsächlich mit den gleichen Voraussetzungen und mit der gleichen Vergütung arbeiten. Der bislang geltende Investitionsabschlag von 5\% für ambulante Leistungen in den Kliniken soll durch das Krankenhausstrukturgesetz entfallen. Ein großer Vorteil sei, dass die ASV nicht budgetiert sei, betonte Munte. „Der Arzt bekommt alle
Die interdisziplinäre Versorgung ist Teil des ASV-Konzepts.

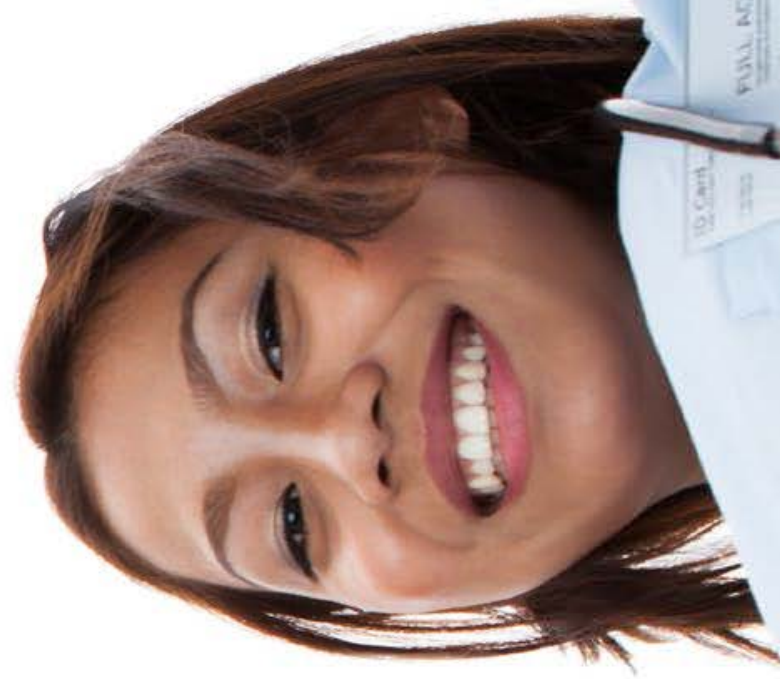

Leistungen bezahlt.“ Die Realisierung der eigenen ASV-Gebührenordnung hält er allerdings für extrem schwierig. „Wir werden die Umsetzung nicht so schnell erleben." Langfristig kann nach seiner Einschätzung die ASV nur mit einer elektronischen Fallakte wirklich funktionieren, damit die beteiligten Behandler alle Daten zeitnah haben. Für ein Problem hält er die „inkonsequente Kooperationsverpflichtung“. Es sei ein Fehler, dass die sektorenübergreifende Kooperation nur in der Onkologie verpflichtend sei, nicht aber in den Indikationen Tuberkulose und Marfan-Syndrom. „Das wird sich hoffentlich noch ändern.“

Nicht immer sektorenübergreifend Auch wenn die Verbindung der Sektoren auf dem Papier stehe, sei sie nicht überall wirksam, sagte er. So gebe es Konstruktionen, in denen Kliniken mit dem eigenen Krankenhaus-MVZ zusammenarbeiten. „Es kann passieren, dass Krankenhaus-dominierte MVZ den ambulanten Bereich darstellen und die Fachärzte in die Minderheit geraten“, warnte er. Positiv bewertete er den beschlossenen Wegfall der „schweren Verlaufsformen“ in der Onkologie und bei Rheuma. Diese Differenzierung sei den Patienten, die damit zum Teil von der neuen Versorgungsform ausgeschlossen würden, nur schwer zu vermitteln.

Ilse Schlingensiepen 